\title{
RINGS WHOSE CYCLIC MODULES ARE DIRECT SUMS OF EXTENDING MODULES
}

\author{
PINAR AYDOĞDU \\ Department of Mathematics, Hacettepe University, 06800 Beytepe, Ankara, Turkey \\ e-mail: paydogdu@hacettepe.edu.tr \\ NOYAN ER \\ Department of Mathematics, University of Rio Grande, Rio Grande, OH 45674, USA \\ e-mail:noyaner@yahoo.com \\ and NIL ORHAN ERTAŞ \\ Department of Mathematics, Karabük University, 78050 Karabük, Turkey \\ e-mail: orhannil@yahoo.com
}

(Received 2 June 2011; revised 27 December 2011; accepted 8 January 2012; first published online 30 March 2012)

\begin{abstract}
Dedekind domains, Artinian serial rings and right uniserial rings share the following property: Every cyclic right module is a direct sum of uniform modules. We first prove the following improvement of the well-known Osofsky-Smith theorem: A cyclic module with every cyclic subfactor a direct sum of extending modules has finite Goldie dimension. So, rings with the above-mentioned property are precisely rings of the title. Furthermore, a ring $R$ is right q.f.d. (cyclics with finite Goldie dimension) if proper cyclic $\left(¥ R_{R}\right)$ right $R$-modules are direct sums of extending modules. $R$ is right serial with all prime ideals maximal and $\cap_{n \in \mathbb{N}} J^{n}=J^{m}$ for some $m \in \mathbb{N}$ if cyclic right $R$-modules are direct sums of quasi-injective modules. A right non-singular ring with the latter property is right Artinian. Thus, hereditary Artinian serial rings are precisely one-sided non-singular rings whose right and left cyclic modules are direct sums of quasi-injectives.
\end{abstract}

2000 Mathematics Subject Classification. Primary 16D10, 16D70, 16P20, 16 P40.

1. Introduction. Our rings are associative with identity, and modules are unitary right modules unless otherwise stated. A module $M$ is called extending if every submodule of $M$ is essential in a direct summand of $M$, and $M$ is called quasi-injective if $M$ is a fully invariant submodule of its injective hull. Quasi-injective modules are extending.

Non-commutative rings whose cyclic (right) modules satisfy injectivity-like properties have been studied in the last four decades since the first non-trivial result in this direction was proved by Osofsky ([16] and [17]): She showed that a ring is semisimple Artinian if its cyclic right modules are injective. Klatt and Levy characterised in [12] commutative rings whose factors are self-injective, a noncommutative analogue of which was studied by Ahsan $([\mathbf{1}, \mathbf{2}])$ and Koehler $([\mathbf{1 3}])$, where they considered rings whose cyclic right (equivalently left) modules are quasi-injective, namely qc-rings. 
The main challenge of Osofsky's result mentioned above is essentially to see that such a ring has finite Goldie dimension. Osofsky and Smith extended this result in the much more general context of extending modules, proving the following ([15]): A cyclic module whose cyclic subfactors are extending has finite Goldie dimension. This result proved to be a master key for verifying some of the results then known as well as proving unknown ones (see [3]).

This paper derives its motivation from the following facts: Neither the extending property, nor quasi-injectivity carries over to finite direct sums (e.g. $\frac{\mathbb{Z}}{p \mathbb{Z}} \oplus \frac{\mathbb{Z}}{p^{3} \mathbb{Z}}$, where $p$ is a prime). Also, rings whose cyclic (right) modules are direct sums of extending (resp. quasi-injective) modules are a proper subclass of rings whose cyclics are extending (resp. quasi-injective)(see Examples 1 and 6). So, the following questions arise naturally:

QuESTION 1. Does the conclusion of the Osofsky-Smith theorem still hold if we assume cyclic subfactors to be direct sums of extending modules?

QUESTION 2. What are the structures of rings with the following properties?

$(\mathrm{P})_{r(l)}$ : Every cyclic right (resp. left) module is a direct sum of extending modules;

$(\mathrm{Q})_{r(l)}$ : Every cyclic right (resp. left) module is a direct sum of quasi-injective modules.

Note that $(\mathrm{Q})_{r}$ implies $(\mathrm{P})_{r}$. Dedekind domains and right uniserial rings satisfy $(\mathrm{P})_{r}$, and Artinian serial rings satisfy $(\mathrm{Q})_{r}$. Clearly $\mathbb{Z}$ is a ring with $(\mathrm{P})_{r}$ but not $(\mathrm{Q})_{r}$. Also, these properties are not left-right symmetric (Examples 2 and 7).

In [4], it is shown that rings all of whose right modules are direct sums of extending modules are precisely rings of finite type and right colocal type (indecomposable right modules have simple socle). That result and its proof constitutes the basis for our key result in this paper. Modifying the proof of [4, Theorem 1], we first obtain the affirmative answer to Question 1 (Theorem 1). Consequently, rings with property $(\mathrm{P})_{r}$ are precisely rings whose cyclic right modules are direct sums of uniform modules (Theorem 2). Although a ring $R$ with property $(\mathrm{P})_{r}$ is not right Noetherian in general, it is when $R$ is a right $\mathrm{V}$ - ring (Example 3 and Corollary 2), or when it is right hereditary (see Remark 1). A right hereditary right V-ring is right Noetherian if and only if every cyclic right module with essential socle is a direct sum of extending modules (Theorem 3). Note that for a ring $R$ to be right q.f.d., it suffices for only proper cyclic $(¥ R)$ right $R$-modules to be direct sums of extending modules (Corollary 9). However, in this case, cyclic right $R$-modules may not be direct sums of uniforms (Example 9).

Next we apply the above-mentioned result to rings satisfying the property $(\mathrm{Q})_{r}$ and obtain the following results: A ring with the property $(\mathrm{Q})_{r}$ is right serial with all prime ideals maximal, and $\cap_{n \in \mathbb{N}} J^{n}=J^{m}$ for some $m \in \mathbb{N}$, where $J$ is the Jacobson radical, which is now nil (see Theorem 4 and its corollaries). A right non-singular ring with property $(\mathrm{Q})_{r}$ is right hereditary, right Artinian and right serial (Corollary 4). It follows that hereditary Artinian serial rings are precisely one-sided non-singular rings satisfying $(\mathrm{Q})_{r}$ and $(\mathrm{Q})_{l}$ (Theorem 5). Here, the assumption $(\mathrm{Q})_{l}$ is not redundant (Example 2). A ring $R$ satisfying $(\mathrm{Q})_{r}$ does not have to be right Noetherian. However, besides the non-singularity case mentioned above, such a ring is right Artinian in each one of the following cases: (i) when $R$ is prime; (ii) when $R$ has the ascending chain 
condition (a.c.c.) on right annihilators (this also improves [2, Theorem 3.3]); or (iii) when $R$ is right perfect (Corollaries 5, 6, and 8). In particular, a ring satisfying $(\mathrm{Q})_{r}$ is right Artinian whenever it is right Noetherian.

In the last section, we study rings whose proper cyclic right modules are direct sums of extending or quasi-injective modules and prove results classifying such rings (Theorems 6 and 7).

2. Preliminaries. Let $R$ be an associative ring with identity and $M$ be a right $R$ module. $M$ is called an extending module if every submodule of $M$ is essential in a direct summand of $M$, equivalently, if every (essentially) closed submodule of $M$ is a direct summand of $M$. Uniform modules are precisely indecomposable extending modules. Extending modules are also known as CS (complements are summands) modules in the literature and have been extensively studied during the last few decades. An extending module is called a quasi-continuous module if the direct sum of any two direct summands is again a direct summand, a continuous module if submodules isomorphic to direct summands are also direct summands. See [3] for a detailed account of these modules and their history. $M$ is called quasi-injective ([11]) if for every submodule $N$ of $M$, every $R$-homomorphism $N \rightarrow M$ can be extended to an $R$-endomorphism of $M$. This is equivalent to $M$ being a fully invariant submodule of its injective hull $E(M)$. The following hierarchy holds for modules: semisimple or injective $\Rightarrow$ quasi-injective $\Rightarrow$ continuous $\Rightarrow$ quasi-continuous $\Rightarrow$ extending. Direct summands of extending (resp. quasi-injective / continuous / quasi-continuous) modules are again extending (resp. quasi-injective / continuous / quasi-continuous), but none of these properties carries over to finite direct sums. For example, $\frac{\mathbb{Z}}{p \mathbb{Z}} \oplus \frac{\mathbb{Z}}{p^{3} \mathbb{Z}}$ is not extending.

A subfactor of $M$ is any submodule of a factor of $M . M$ is called a q.f.d. module if all factors of $M$ have finite Goldie dimension. $R$ is called a right q.f.d. ring if $R_{R}$ is a q.f.d. module. $R$ is called a right V-ring (after Villamayor) if simple right $R$-modules are injective. If $M$ is a submodule of a direct sum $\bigoplus_{i \in I} A_{i}$ of modules $A_{i}$ and $\pi_{j}$ : $\bigoplus_{i \in I} A_{i} \rightarrow A_{j}$ are the canonical projections, then $\operatorname{supp}_{\bigoplus_{i \in I}} A_{i}(M)=\left\{i \in I: \pi_{i}(M) \neq 0\right\}$ is the support of $M$ in $\bigoplus_{i \in I} A_{i}$. For a module $M$, a family of submodules $\left\{N_{i}: i \in I\right\}$ of $M$ (or their sum) is called a local summand of $M$ if their sum is direct and $\bigoplus_{i \in F} N_{i}$ is a direct summand of $M$ for every finite subset $F$ of $I$.

For a module $M, \operatorname{rad}(M), \operatorname{soc}(M), Z(M)$ stand for the Jacobson radical, the socle, and the singular submodule of $M$, respectively. We will denote the Jacobson radical of a ring $R$ by $J$.

For definitions and properties of standard concepts used throughout the paper, the reader is referred to [6] and [14].

3. An extension of the Osofsky-Smith Theorem. In this section, Question 1 is answered affirmatively. To this end, we modify the proof of [4, Theorem 1]. It is important to note that this modification is made, and this section is structured, with a view to also address the situation in Theorem 3 (also see the remark following Lemma 2).

Lemma 1. Let $A$ be a cyclic module. If every cyclic subfactor of $A$ is a direct sum of extending modules, then either $A$ is q.f.d. or there exists a cyclic extending subfactor $N$ of $A$ with infinite essential socle, which is a local summand of $N$. 
Proof. Assume $A$ is not q.f.d. Then the result follows by the same argument as in the first paragraph of the proof of [4, Theorem 1] replacing $R$ with $A$.

LEMMA 2. Let $C$ be a module with infinite essential socle, which is a local summand of $C$, and $\pi: C \rightarrow \frac{C}{\operatorname{soc}(C)}$ be the natural projection. Assume that $C$ has an essential submodule $D=\bigoplus_{n \in \mathbb{N}} D_{n}$ where no $D_{n}$ is semisimple. Then, a cyclic submodule $B$ of $C$ cannot simultaneously satisfy the conditions

(i) $B$ is a direct sum of extending modules, and

(ii) $\pi(B) \cap \pi(D)$ is essential in $\pi(D)$ and $\pi(B)$.

Proof. Assume, contrarily, that $C$ has a cyclic submodule $B$ satisfying the conditions ( $i$ ) and (ii) above. Put, for any submodule $X$ of $C, \bar{X}=\pi(X)$. Also let $S_{n}=\operatorname{soc}\left(D_{n}\right)$ for each $n \in \mathbb{N}$. Now, for all $n \in \mathbb{N} \bar{B} \cap \overline{D_{n}} \neq 0$. This means that $(\operatorname{soc}(C)+B) \cap\left(D_{n}+\operatorname{soc}(C)\right)=\operatorname{soc}(C)+\left(B \cap\left(D_{n}+\operatorname{soc}(C)\right)\right)$ is not semisimple. Put $Z_{n}=B \cap\left(D_{n}+\operatorname{soc}(C)\right)$. Then $Z_{n}$ is not semisimple. There exists some submodule $K$ of $\operatorname{soc}(C)$ such that $D_{n}+\operatorname{soc}(C)=D_{n} \oplus K$. If we had $B \cap D_{n}=Z_{n} \cap D_{n}=0, Z_{n}$ could be embedded in $K$, whence it would be semisimple, contradicting the above observation. Therefore, $Z_{n} \cap D_{n}=B \cap D_{n} \neq 0$. In particular, $B \cap S_{n} \neq 0$. This implies that $\operatorname{supp}_{\bigoplus_{n \in \mathbb{N}}} S_{n}(\operatorname{soc}(B))$ is infinite.

Now we follow the last part of the proof of [4, Theorem 1] verbatim: By assumption, $B=B_{1} \oplus \ldots \oplus B_{n}$ for some extending modules $B_{k}$. By the preceding paragraph, there exists some $i \in\{1, \ldots, n\}$ such that $\operatorname{supp}_{\bigoplus_{n \in \mathbb{N}} S_{n}} \operatorname{soc}\left(B_{i}\right)$ is infinite. In particular, $B_{i}$ is not semisimple. Since $B_{i}$ is extending, without loss of generality, we can assume $B_{i}$ has countably generated socle and write $\operatorname{soc}\left(B_{i}\right)=\bigoplus_{n \in \mathbb{N}} T_{n}$, where $T_{n}$ are simple modules. Now, we can select, inductively, a subsequence of distinct indices $\left(n_{k}\right)_{k \in \mathbb{N}}$ such that

$$
\max \left(\operatorname{supp}_{\bigoplus_{n \in \mathbb{N}} S_{n}}\left(T_{n_{k+1}}\right)\right)>\max \left(\operatorname{supp}_{\bigoplus_{n \in \mathbb{N}} S_{n}}\left(T_{n_{k}}\right)\right) \text {, for each } k \in \mathbb{N} \text {. }
$$

In particular, $\max \left(\operatorname{supp}_{\oplus_{n \in \mathbb{N}} S_{n}}\left(T_{n_{k+1}}\right)\right)>k$, for each $k \in \mathbb{N}$. Since $B_{i}$ is extending, we can find a direct summand $U$ of $B_{i}$ such that $\bigoplus_{k \in \mathbb{N}} T_{n_{k}}$ is essential in $U$. Since $U$ is cyclic, $U \neq \bigoplus_{k \in \mathbb{N}} T_{n_{k}}$. Now we will see that $U \cap D$ is semisimple. In order to do that, it suffices to verify that for each $l \in \mathbb{N} U \cap \bigoplus_{i=1}^{l} D_{i}$ is semisimple. First we claim that $U \cap \bigoplus_{i=1}^{l} S_{i} \subseteq \bigoplus_{k=1}^{l+1} T_{n_{k}}$ for each $l \in \mathbb{N}$ :

Fix $l \in \mathbb{N}$ and assume the contrary of the above claim. Then there exists some $x=t_{n_{1}}+\cdots+t_{n_{m}} \in U \cap \bigoplus_{i=1}^{l} S_{i}$ with $t_{n_{k}} \in T_{n_{k}}, t_{n_{m}} \neq 0$, and $m>l+1$. $t_{n_{m}}$ generates $T_{n_{m}}$. Thus, we have

$$
\begin{aligned}
& \max \left(\operatorname{supp}_{\bigoplus_{n \in \mathbb{N}} S_{n}}\left(T_{n_{m}}\right)\right)=\max \left(\operatorname{supp}_{\bigoplus_{n \in \mathbb{N}} S_{n}}\left(t_{n_{m}}\right)\right)>\max \left(\operatorname{supp}_{\bigoplus_{n \in \mathbb{N}} S_{n}}\left(T_{n_{m-1}}\right)\right)= \\
& \max \left(\operatorname{supp}_{\bigoplus_{n \in \mathbb{N}} S_{n}}(Q)\right),
\end{aligned}
$$

where $Q=\left(\bigoplus_{i=1}^{l} S_{i}\right)+\left(\bigoplus_{j=1}^{m-1} T_{n_{j}}\right)$. This is a contradiction, since $t_{n_{m}} \in Q$. This proves the claim above, implying that $\operatorname{soc}\left(U \cap \bigoplus_{i=1}^{l} D_{i}\right)=U \cap \bigoplus_{i=1}^{l} S_{i}$ is a finitely generated (and essential) submodule of $U \cap \bigoplus_{i=1}^{l} D_{i}$. Hence, $\operatorname{soc}\left(U \cap \bigoplus_{i=1}^{l} D_{i}\right)=U \cap \bigoplus_{i=1}^{l} D_{i}$. Therefore, $U \cap D$ must be semisimple.

Recall that $U$ is a non-semisimple submodule of $B$, and $\bar{B} \cap \bar{D}$ is essential in both $\bar{B}$ and $\bar{D}$. Hence, $\bar{U}$ is a non-zero submodule of $\bar{B}$ and thus, it must have non-zero intersection with $\bar{D}$. But, this contradicts the semisimplicity of $U \cap D$. Now the result follows. 
Notice that the module $C$ in Lemma 2 is not assumed to be cyclic, which would have been sufficient for the purposes of Theorem 1 . This makes the lemma useful not only in this section, but for proving Theorem 3 in the next section as well.

THEOREM 1. Let $A$ be a cyclic module such that every cyclic subfactor of $A$ is a direct sum of extending modules. Then $A$ is a q.f.d. module.

Proof. Assume, contrarily, that $A$ is not q.f.d. By Lemma 1, we can find a cyclic extending subfactor $N$ of $A$ with infinite essential socle, which is also a local summand of $N$. Then by our assumption, $\frac{N}{\operatorname{soc}(N)}$ has a non-zero extending direct summand $\frac{N^{\prime}}{\operatorname{soc}(N)}$. In particular, $N^{\prime}$ is a non-semisimple submodule of $N$. We can write $N^{\prime}=M+\operatorname{soc}(N)$ for some cyclic and non-semisimple submodule $M$ of $N$. Clearly, $\operatorname{soc}(M)$ is infinitely generated and essential in $M$ as well a local summand in $M$. Furthermore, $\frac{M}{\operatorname{soc}(M)} \cong$ $\frac{N^{\prime}}{\operatorname{soc}\left(N^{\prime}\right)}$ is also extending. Again by assumption we have $M=M_{1} \oplus \ldots \oplus M_{n}$ for some extending submodules $M_{n}$ of $M$. There must be some $k_{0}$ such that $C=M_{k_{0}}$ is not semisimple. So now, $C$ is a cyclic extending module with infinite essential socle, which is a local summand, and $\frac{C}{\operatorname{soc}(C)}$, being isomorphic to a direct summand of $\frac{M}{\operatorname{soc}(M)}$, is also extending. Now we can write $\operatorname{soc}(C)=\bigoplus_{n \in \mathbb{N}} S_{n}$, where $S_{n}$ are infinitely generated semisimple modules. Since $C$ is cyclic extending, each $S_{n}$ is essential and proper in a direct summand $D_{n}$ of $C$. Then set $D=\bigoplus_{n \in \mathbb{N}} D_{n}$. Since $\frac{C}{\operatorname{soc}(C)}$ is also extending, $\frac{D}{\operatorname{soc}(C)}$ is essential in a direct summand $\frac{Y}{\operatorname{soc}(C)}$ of $\frac{C}{\operatorname{soc}(C)}$ for some submodule $Y$ of $C$. There exists a cyclic submodule $B$ of $Y$ such that $Y=B+\operatorname{soc}(C)$. This is a contradiction by Lemma 2.

COROllary 1. (Ososfky-Smith) A cyclic module with every cyclic subfactor extending is q.f.d.

4. Rings whose cyclics are direct sums of extending modules. Dedekind domains, Artinian serial rings and right uniserial rings share the property that cyclic right modules are direct sums of uniform modules. The next result characterises rings having this property as those whose cyclic right modules are direct sums of extending modules, giving an answer to the first part of Question 2 (see Introduction). It follows immediately from Theorem 1 and the fact that uniform modules are quasi-continuous.

THEOREM 2. The following are equivalent for a ring $R$ :

(i) $R$ satisfies $(P)_{r}$,

(ii) Every cyclic right $R$-module is a direct sum of quasi-continuous modules,

(iii) Every cyclic right $R$-module is a direct sum of uniform modules.

In particular, a ring satisfying $(P)_{r}$ is a right q.f.d. ring.

In Theorem 2 (ii), the assumption quasi-continuous cannot be replaced by continuous since, for example, the ring $\mathbb{Z}$ satisfies the conditions of Theorem 2 , but it is not a continuous module over itself.

The following example distinguishes rings satisfying the property $(\mathrm{P})_{r}$ from those whose cyclic right modules are extending.

EXAMPLE 1 . Let $R$ be any Artinian serial ring such that $R_{R}=\bigoplus_{i=1}^{n} e_{i} R$, where $n>1, e_{i} R$ are uniserial, $e_{1} R \cong e_{2} R$, and $\operatorname{cl}\left(e_{1} R\right)=3$ (see [18, 8.32]). Then $e_{1} R$ has a composition series $0 \subset V \subset U \subset e_{1} R$. Then, by [3, Lemma 7.4], the module $M=$ $e_{1} R \oplus \frac{U}{V}$ is not extending. Now $\frac{U}{V}$ is isomorphic to either $\frac{e_{2} R}{e_{2} J}$ or $\frac{e_{i} R}{e_{i} J}$ for some $i \neq 1,2$, 
say $i=3$. So, $M \cong e_{1} R \oplus \frac{e_{2} R}{e_{2} J}$ or $M \cong e_{1} R \oplus \frac{e_{3} R}{e_{3} J}$. In either case, $M$ is a cyclic module. This shows that $R$ has a cyclic module which is not extending. However, $R$ satisfies $(\mathrm{P})_{r}$ and $(\mathrm{P})_{l}$ since it is Artinian serial.

The property of a ring that its cyclic modules are direct sums of extending modules is not left-right symmetric:

EXAMPLE 2. Let $\mathbb{Q}$ and $\mathbb{R}$ denote the sets of rational numbers and real numbers,

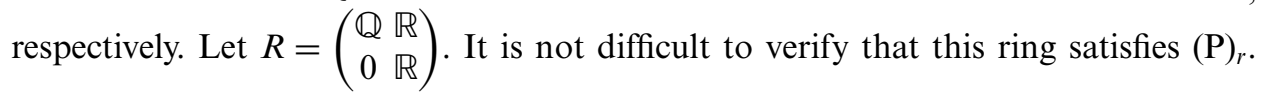
However, $R e_{22}$ has infinite Goldie dimension. So, $R$ does not satisfy $(\mathrm{P})_{l}$.

For a ring with $(\mathrm{P})_{r}$ to be right Noetherian, an additional condition is necessary.

EXAMPLE 3. A right uniserial ring obviously satisfies $(\mathrm{P})_{r}$, but it does not have to be right Noetherian.

COROLlaRY 2. A right $V$-ring $R$ with property $(P)_{r}$ is right Noetherian.

Proof. Suppose that $S$ is a semisimple module, which is not injective. Then, pick some $x \in E(S)-S . x R$ must have infinite socle. But this is not possible because of Theorem 1. Thus, all semisimple right modules over $R$ are injective, proving that $R$ is right Noetherian.

REMARK 1. One can see, either from Theorem 2 or from [3, Theorem 10.5] that a right hereditary ring with property $(\mathrm{P})_{r}$ is right Noetherian as well. No other pair of the properties right hereditary, right Noetherian, and $(\mathrm{P})_{r}$ imply the third one, as is shown by the next two examples.

EXAMPLE 4. Let $R$ be any Artinian serial ring which is not right (equivalently left) non-singular. Then $R$ satisfies $(\mathrm{P})_{r}$ and $(\mathrm{P})_{l}$, but it is neither right hereditary, nor right $\mathrm{V}$.

EXAmPLE 5. Let $R=\left(\begin{array}{cc}K & K^{2} \\ 0 & K\end{array}\right)$. Then, $R$ is Artinian and right hereditary. However, the first row is not an extending right $R$-module. Thus, $R$ does not satisfy $(\mathrm{P})_{r}$.

THEOREM 3. Let $R$ be a right hereditary and right $V$-ring. The following are equivalent:

(i) $R$ is right Noetherian,

(ii) Every cyclic right $R$-module with essential socle is a direct sum of extending modules.

Proof. One direction is clear. Now assume the condition (ii) and, contrarily, that $R$ is not right Noetherian. Then there exists a non-injective semisimple module $S$. So, we can pick some $x \in E(S)-S$. Clearly, $x R$ has infinite socle. By assumption $x R$ has an extending direct summand $A$ with infinite socle. As in Theorem 1, we can write $\operatorname{soc}(A)=\bigoplus_{n \in \mathbb{N}} S_{n}$, where $S_{n}$ are infinitely generated semisimple modules, and since $A$ is extending, each $S_{n}$ is essential and proper in some direct summand $D_{n}$ of $A$. Call $D=\bigoplus_{n \in \mathbb{N}} D_{n}$. Now let $\pi: E(A) \rightarrow \frac{E(A)}{\operatorname{soc}(A)}$ be the obvious projection. For any submodule $P$ of $E(A)$ containing $\operatorname{soc}(A)$, put $\bar{P}=\frac{P}{\operatorname{soc}(A)}$. Since $R$ is right hereditary $\bar{E}$ is injective. $\bar{A}$ is essential in a direct summand $X$ of $\bar{E}$. Then, there is a decomposition $X=Y \oplus Y^{\prime}$, where $\bar{D}$ is essential in $Y$. Consider the obvious projection $\pi_{Y}: Y \oplus Y^{\prime} \rightarrow Y$. Since $\bar{A} \cap Y \subseteq \pi_{Y}(\bar{A})$ and $\bar{A} \cap Y$ is essential in $Y$, then so is $\pi_{Y}(\bar{A})$. Thus, $Y$ essentially contains a cyclic module. So, let $L$ be any cyclic essential 
submodule of $Y$. There exists some cyclic submodule $B$ of $E(A)$ such that $\pi(B)=L$. Taking $C=E(A)$ in Lemma 2, this yields a contradiction.

5. Rings whose cyclics are direct sums of quasi-injectives. Ahsan $([1,2])$ and Koehler ([13]) studied qc-rings, namely rings with every cyclic right module quasiinjective. In [13], Koehler characterised such rings as (finite) direct sums of rings which are either semisimple Artinian, or rank 0 maximal valuation duo. Since the direct sum of two quasi-injective modules is not necessarily quasi-injective (see Introduction), it is interesting to study rings satisfying the property $(\mathrm{Q})_{r}$.

Any Artinian serial ring clearly satisfies both $(\mathrm{Q})_{r}$ and $(\mathrm{Q})_{l}$. The qc condition is left-right symmetric (see [13]). Rings satisfying $(\mathrm{Q})_{r}$ form a larger class than qc-rings (Example 6 below), and (Q), does not imply (Q) $)_{l}$ (Example 7):

EXAMPLE 6. Let $R$ be a hereditary Artinian serial ring which is not semisimple. Then $R$ is not a qc-ring: Let $R=\bigoplus_{i=1}^{n} e_{i} R$, where $e_{i}$ form a complete set of orthogonal primitive idempotents. By assumption, there is some $i \in\{1, \ldots, n\}$ such that $e_{i} R$ is not a simple right ideal. Now let $S=\operatorname{soc}\left(e_{i} R\right) . S$ is a homomorphic image of $e_{j} R$ for some $j \in\{1, \ldots, n\}$. Since $R$ is right non-singular and $e_{j} R$ is uniserial, this forces $e_{j} R$ to be simple. In particular, $i \neq j$. Hence, $e_{i} R \oplus e_{j} R$ is a direct summand of $R_{R}$, and it cannot be quasi-injective. So, $R$ satisfies the properties $(\mathrm{Q})_{r}$ and $(\mathrm{Q})_{l}$, but it is not a qc-ring.

The next theorem describes rings with property $(\mathrm{Q})_{r}$. In [9], rings whose right ideals are finite direct sums of quasi-injective right ideals are called right $\Sigma-q$ rings.

THEOREM 4. Let $R$ be a ring with property $(Q)_{r}$. Then $R$ is a right serial ring in the form $R=\left(\begin{array}{cc}f R f & f R e \\ 0 & e R e\end{array}\right)$, where $e$ Re and $f R f$ are rings with $(Q)_{r}$, eRe is a right hereditary, right Artinian, right $\Sigma-q$ ring, and $Z(f R)$ is essential in $f R$.

Proof. Assume that $R$ satisfies the property (Q) $r$. Then, by Theorem 2, $R_{R}$ is a direct sum of uniform right ideals. Thus, we can write $R_{R}=$ $e_{1} R \oplus \ldots \oplus e_{n} R \oplus f_{1} R \oplus \ldots \oplus f_{m} R, \quad$ where $\quad\left\{e_{i}: i=1, \ldots, n\right\} \cup\left\{f_{t}: t=1, \ldots, m\right\}$ is a complete set of orthogonal primitive idempotents, $e_{i} R$ are non-singular and $Z\left(f_{t} R\right) \neq 0$. Now let $g \in\left\{e_{i}: i=1, \ldots, n\right\} \cup\left\{f_{t}: t=1, \ldots, m\right\}$. Since $g R$ is uniform quasi-injective, $\operatorname{End}(g R) \cong g R g$ is a local ring and $g R$ is a local module. By assumption of the $(\mathrm{Q})_{r}$ condition, all non-zero factors of $g R$ must be local modules which are direct sums of quasi-injectives. But this means that all non-zero factors of $g R$ are uniform, which implies that $g R$ must be uniserial. Therefore, $R$ is a right serial ring.

Claim. For the above choice of $g, g R$ contains no infinite properly ascending or descending chains of cyclic right ideals which are not singular.

Let $\left(A_{k}\right)_{k \in \mathbb{N}}$ be such a chain. Then each $A_{k}$ is isomorphic to one of $e_{i} R$ or $f_{t} R$, which are only finitely many. Since $e_{i} R$ and $f_{t} R$ are quasi-injective and our chain is properly ascending or descending, we get a contradiction, proving our claim.

Let $A$ be any right ideal in $g R$ which is not singular. Then we can inductively pick elements $a_{k}(k \in \mathbb{N})$ such that $a_{1} \in A-Z(A)$, and $a_{k+1} \in A-a_{k} R$. By the above arguments, the process will stop when $a_{k} R=A$. So $A$ is cyclic. Furthermore, since there are no singular right ideals between $g R$ and $A, \frac{g R}{A}$ has finite length. Hence, $A=g R$ or $A=g J^{m}$ for some $m$. Moreover, the above claim still holds without the cyclic assumption.

The above arguments imply that each $e_{i} R$ has finite length. 
Now put $e=\sum_{i=1}^{n} e_{i}$ and $f=\sum_{t=1}^{m} f_{t}$. Since $f R$ has essential singular submodule and $e R$ is non-singular, $0=\operatorname{Hom}_{R}(f R, e R) \cong e R f$. Since $\frac{R}{f R e} \cong e R e \times f R f$ and $(\mathrm{Q}) r$ is inherited by factor rings, $e R e$ and $f R f$ satisfy $(\mathrm{Q})_{r}$. Clearly, $e R e$ is now a right Artinian, right serial and right non-singular ring. Then, by [5, Theorem 5.16], eRe is right semihereditary, whence right hereditary since it is right Artinian. Now, any right ideal $I$ of this ring is a direct sum of right ideals which can be embedded in the principle indecomposable right ideals. Hence, $I$ is a finite direct sum of quasi-injective right ideals. So, $e R e$ is a right $\Sigma-q$ ring. This concludes the proof.

COROLlaRY 3. If $R$ satisfies $(Q)_{r}$, then $\frac{R}{Z\left(R_{R}\right)}$ is a right Artinian ring.

Proof. Let $e$ be a primitive idempotent of $R$. By the proof of Theorem 4, $e R$ does not contain an infinite properly descending chain of (cyclic) right ideals, which are not singular. This immediately yields that $\frac{e R}{Z(e R)}$ is an Artinian right $R$-module. Now the result follows.

The qc-rings are serial (see [13]). However, as the next example shows, rings with property $(\mathrm{Q})_{r}$ do not have to be left serial, and for this reason and by Theorem 4, they do not have to satisfy $(\mathrm{Q})_{l}$ either. In [7], Fuller investigated rings of finite type with every indecomposable right (resp. left) module quasi-injective and called them rings of right (resp. left) invariant module type.

EXAMPLE 7. Let $R$ be a ring with right but not left invariant module type. $R$ is a right serial ring satisfying $(\mathrm{Q})_{r}$, but it is not left serial, and does not satisfy $(\mathrm{Q})_{l}$. Otherwise, it would then be Artinian serial, in which case all right modules and left modules would be direct sums of quasi-injective modules.

Also note that since qc-rings are not necessarily (right) Noetherian (see [13]), neither are rings satisfying the property $(\mathrm{Q})_{r}$.

COROLLARY 4. A right non-singular ring $R$ satisfying $(Q)_{r}$ is right hereditary, right Artinian and right serial. In particular, $R$ is a right $\Sigma-q$ ring.

Such a ring as in Corollary 4 is furthermore isomorphic to a finite product of certain upper triangular matrix rings shown in [9, Theorem 23].

COROLLARY 5. The following conditions hold for a ring $R$ satisfying $(Q)_{r}$ :

(i) If $R$ is prime then it is Artinian.

(ii) Prime ideals of $R$ are maximal. In particular, $J$ is nil.

Proof. (i) It can be seen, by using the same argument as in [8, Theorem 2.2], that $R$ is right non-singular. Then, it follows by Corollary 4 that $R$ is Artinian.

(ii) Any prime factor of $R$ also satisfies the property $(\mathrm{Q})_{r}$, hence it is simple Artinian by part (i). Thus, prime ideals of $R$ are maximal, whence primitive. In particular, the prime radical of $R$ coincides with $J$. So, $J$ is nil.

Ahsan proved in [2, Theorem 3.3] that if $R$ is a qc-ring with a.c.c. on right annihilators then $J$ is nilpotent. The next corollary improves this result.

COROllary 6. A ring $R$ with $(Q)_{\text {r }}$ and a.c.c on right annihilators is right Artinian. In particular, a right Noetherian ring with $(Q)_{r}$ is right Artinian.

Proof. In this case, $Z\left(R_{R}\right)$ is nilpotent. Now let $\left\{e_{i}: i=1, \ldots, s\right\}$ be a complete set of primitive orthogonal idempotents of $R$. Fix $e_{i}$. By Corollary $3, \frac{e_{i} R}{Z\left(e_{i} R\right)}$ has finite 
length. This implies that $Z\left(e_{i} R\right)=e_{i} J^{t_{i}}$ for some $t_{i}$. Let $t=\max \left\{t_{i}: i=1, \ldots, s\right\}$. Then, each $e_{i} J^{t}$ is nilpotent. Then $J^{t}=\bigoplus_{i=1}^{s} e_{i} J^{t}$ is nilpotent, whence so is $J$. So, $R$ is a semiprimary ring with all cyclic right $R$-modules having finite Goldie dimension. This means that every cyclic right module has finitely generated essential socle. Hence, $R$ is right Artinian.

COROLlaRY 7. If R satisfies property $(Q)_{r}$, then $\bigcap_{n \in \mathbb{N}} J^{n}=J^{m}$ for some $m$.

Proof. Let $e$ be a primitive idempotent of $R$. Now, one can use the same argument as in the paragraph preceding [5, Lemma 5.18] for a right serial ring (instead of a serial one) to see that $\frac{e R}{\bigcap_{n \in \mathbb{N}} e J^{n}}=\frac{e R}{e\left(\bigcap_{n \in \mathbb{N}} J^{n}\right)}$ is a Noetherian right $R$-module. Then $\frac{R}{\bigcap_{n \in \mathbb{N}} J^{n}}$ is right Noetherian with $(\mathrm{Q})_{r}$, whence it is right Artinian by Corollary 6. Now the result follows.

COROLlary 8. A right perfect ring $R$ satisfying $(Q)_{r}$ is right Artinian.

Proof. By Corollary $7, J^{m}=J^{m+1}=\ldots$ for some $m$. Recall that since $R$ is semilocal, $\operatorname{rad}(M)=M J$ for any module $M$. Also by right perfect assumption every non-zero module has proper radical. This means that $J^{m}=0 . R$ is right Artinian by the same argument as in the proof of Corollary 7 (or by the one at the end of Corollary 6).

THEOREM 5. The following conditions are equivalent for any ring $R$ :

(i) $R$ is hereditary Artinian serial,

(ii) $R$ is a right or left non-singular ring satisfying $(Q)_{r}$ and $(Q)_{l}$.

(iii) $R$ is a right serial, right or left non-singular ring satisfying $(Q)_{l}$.

(iv) $R$ is a left serial, right or left non-singular ring satisfying $(Q)_{r}$.

Proof. $(i) \Rightarrow($ ii $)-($ iv $)$ are obvious.

$($ ii $) \Rightarrow(i)$ Let $R$ be right non-singular ring satisfying $(\mathrm{Q})_{r}$ and $(\mathrm{Q})_{l}$. Then $R$ is serial and right Artinian by Theorem 4 and Corollary 4, whence it is Artinian. Since by [5, Corollary 5.12] $R$ is right and left non-singular, now the result follows by Corollary 4 . The proof for left non-singular case is symmetric.

(iii) $\Rightarrow$ (i) $R$ is serial by assumption and Theorem 4 . As in the proof of $(i i) \Rightarrow(i), R$ is both right and left non-singular, whence left hereditary and left Artinian by Corollary 4. Now $R$ is Artinian, and the result follows by seriality of $R$ and [5, Theorem 5.16]. And the proof of $(i v) \Rightarrow(i)$ is symmetric. Now the theorem follows.

The following example shows that, in Theorem 5, the assumptions of $(\mathrm{Q})_{l}$ to get (ii) $\Rightarrow(i)$, and of left serial to get $(i v) \Rightarrow(i)$, are not redundant:

EXAMPLE 8. Let

$$
R=\left(\begin{array}{ccc}
\frac{\mathbb{Z}}{2 \mathbb{Z}} & 0 & \frac{\mathbb{Z}}{2 \mathbb{Z}} \\
0 & \frac{\mathbb{Z}}{2 \mathbb{Z}} & \frac{\mathbb{Z}}{2 \mathbb{Z}} \\
0 & 0 & \frac{\mathbb{Z}}{2 \mathbb{Z}}
\end{array}\right) .
$$

$R$ is right but not left serial, right hereditary with $J^{2}=0$, and indecomposable right $R$-modules are uniform (see [19, Example 2]). Now [19, Theorem 3.6] applies to yield that every right $R$-module is a direct sum of uniform modules. It is easy to see that every factor of each $e_{i i} R(i \in\{1,2,3\})$ is quasi-injective. Moreover, since a uniform right $R$-module is a sum of factors of $e_{i i} R$, uniforms are quasi-injective as well. So, $R$ satisfies $(\mathrm{Q})_{r}$, but not $(\mathrm{Q})_{l}$. 
We will conclude this section with a sufficient condition for a ring to satisfy $(\mathrm{Q})_{r}$. A module $M$ is said to be completely quasi-injective if every factor of $M$ is quasi-injective. We need the following lemma, which may be known.

LEMMA 3. Let $R$ be a right hereditary ring. Then any projective quasi-injective module is completely quasi-injective.

Proof. Let $B$ be a quasi-injective and projective module over a right hereditary ring $R$. Let $X$ be any submodule of $B$.

Claim. $\frac{B}{X}$ is a fully invariant submodule of $\frac{E(B)}{X}$.

Let $F \in \operatorname{End}\left(\frac{E(B)}{X}\right)$ be any endomorphism. Put $f=F_{\mid \frac{B}{X}}$. Let $\pi: B \rightarrow \frac{B}{X}$ and $\pi^{\prime}$ : $E(B) \rightarrow \frac{E(B)}{X}$ be the obvious projections. Since $B$ is projective, there is a map $h: B \rightarrow$ $E(B)$ such that $\pi^{\prime} h=f \pi$. Since $B$ is a quasi-injective module, it is fully invariant in $E(B)$, so that $h(B)$ is contained in $B$. But then, $f\left(\frac{B}{X}\right)=\pi^{\prime} h(B)$ is contained in $\frac{B}{X}$. This proves our claim.

Now, since $R$ is right hereditary, $\frac{E(B)}{X}$ is injective, and thus it contains an injective hull $T$ of $\frac{B}{X}$ containing $\frac{B}{X}$. It is now clear that $\frac{B}{X}$ is a fully invariant submodule of $T$. Therefore, $\frac{B}{X}$ is quasi-injective, proving the lemma.

PROPOSITION 1. Let $R$ be a right hereditary and (2-sided) serial ring such that $R=\bigoplus_{i=1}^{n} e_{i} R$, where $e_{i} R$ are quasi-injective and uniserial right ideals. Then, $R$ satisfies the property $(Q)_{r}$. In fact, all finitely generated right modules are direct sums of quasiinjectives. In particular, $R$ is a right $\Sigma$-q ring.

Proof. First note that, in this case, $R$ is right Noetherian. Let $A$ be a finitely generated right $R$-module. It is well-known that over a serial ring every finitely presented module is serial. Hence, $A=A_{1} \oplus \ldots \oplus A_{n}$, where $A_{i}$ are cyclic uniserial modules. Then, each $A_{i}$ is an epimorphic image of some $e_{j} R . e_{j} R$ is completely quasi-injective by Lemma 3 . Therefore, $A_{i}$ is quasi-injective for $i=1, \ldots, n$. This concludes the proof.

We end this section with an observation: We have seen that $(\mathrm{P})_{r}$ is a weaker condition than $(\mathrm{Q})_{r}$. Consider the following condition:

$(\mathrm{C})_{r}$ : Every cyclic (right) module is a direct sum of continuous modules.

So, we now have the hierarchy $(\mathrm{Q})_{r} \Rightarrow(\mathrm{C})_{r} \Rightarrow(\mathrm{P})_{r}$, and by the remark following Theorem 2, the class of rings satisfying $(\mathrm{P})_{r}$ is larger than the class of rings with $(\mathrm{C})_{r}$. We do not know whether $(\mathrm{Q})_{r}$ is equivalent to $(\mathrm{C})_{r}$ or not. However, one can easily verify that Theorems 4 and 5, and Corollaries 3, 4, 6, 7 and 8 still hold, with the exception of statements about $\Sigma$-q rings, if $(\mathrm{Q})_{r}$ is replaced by $(\mathrm{C})_{r}$.

6. Rings whose proper cyclics are direct sums of extending or quasi-injective modules. In [10], Jain, Singh and Symonds considered rings $R$ whose proper cyclic right modules (that is, cyclic right modules not isomorphic to $R_{R}$ ) are quasi-injective, and called such rings right PCQI. Dedekind domains are PCQI-rings not satisfying $(\mathrm{Q})_{r}$ in general. On the other hand, Artinian serial rings are not necessarily PCQI, but they satisfy $(\mathrm{Q})_{r}$. So, these two types of rings both belong to the larger class of rings whose proper cyclic (right) modules are direct sums of quasi-injective (hence extending) modules. Also, there exist rings which do not satisfy $(\mathrm{P})_{r}$ and over which proper cyclic modules are direct sums of extending modules (see Example 9). 
Motivated by these arguments, in this section, we will consider the following conditions:

$\left(\mathrm{P}^{\prime}\right)_{r}$ : Every proper cyclic right $R$-module is a direct sum of extending modules.

$\left(\mathrm{Q}^{\prime}\right)_{r}$ : Every proper cyclic right $R$-module is a direct sum of quasi-injective modules.

We will show that, similar to a ring with property $(\mathrm{P})_{r}$, a ring with property $\left(\mathrm{P}^{\prime}\right)_{r}$ is also right q.f.d. However, rings with $\left(\mathrm{P}^{\prime}\right)_{r}$ do not have to satisfy the property $(\mathrm{P})_{r}$ (Example 9). In other words, in view of Theorem 2, over a ring with $\left(\mathrm{P}^{\prime}\right)_{r}$, it is not necessarily true that every cyclic right module is a direct sum of uniform modules.

THEOREM 6. Let $R$ be a ring satisfying the property $\left(P^{\prime}\right)_{r}$. Then, either

(i) $R$ satisfies the property $(P)_{r}$, or

(ii) $R_{R}$ is indecomposable and $R$ is a right q.f.d. ring with udim $\left(R_{R}\right)>1$. Hence, in this case, $\frac{R}{I}$ is a right uniform ring for any non-zero nil ideal $I$. In particular, either $R$ is semiprime (resp. $\left.\operatorname{soc}\left(R_{R}\right)=0\right)$ or $\frac{R}{N}\left(\operatorname{resp} \cdot \frac{R}{\operatorname{soc}\left(R_{R}\right)}\right)$ is a right uniform ring, where $N$ is the prime radical of $R$.

Proof. Suppose $R$ has property $\left(\mathrm{P}^{\prime}\right)_{r}$. Then for every essential right ideal $I$ of $R,\left(\frac{R}{I}\right)_{R}$ has the property that every cyclic subfactor is a direct sum of extending modules. Then by Theorem $1,\left(\frac{R}{I}\right)_{R}$ has finite uniform dimension. This implies by [3, Lemma 5.14] that $\frac{R}{\operatorname{soc}\left(R_{R}\right)}$ has finite (right) uniform dimension as well. Now we will see that $\operatorname{soc}\left(R_{R}\right)$ is finitely generated: Assume the contrary. Then we can write $\operatorname{soc}\left(R_{R}\right)=\bigoplus_{i \in \Gamma} V_{i}$, where $V_{i}$ are infinitely generated semisimple right ideals and $|\Gamma|=\infty$. If, for infinitely many $i \in \Gamma, V_{i}$ were right ideals having proper essential extensions in $R_{R}$, this would contradict the fact that $\frac{R}{\operatorname{soc}\left(R_{R}\right)}$ has finite uniform dimension. Thus, $V_{i}$ are closed right ideals of $R$ for all but finitely many $i \in \Gamma$.

Now pick any $i \in \Gamma$ such that $V_{i}$ is closed in $R_{R}$. Then $V_{i}$ is not a direct summand of $R_{R}$, whence $\frac{R}{V_{i}}=\bigoplus_{k=1}^{n} \frac{A_{k}}{V_{i}}$, where $\frac{A_{k}}{V_{i}}$ are extending modules by assumption.

Let $\frac{X}{V_{i}}$ be a simple submodule of $\frac{R}{V_{i}}$. Since $V_{i}$ is closed in $R_{R}, X$ is not an essential extension of $V_{i}$, yielding that $X=V_{i} \oplus T$ for some simple submodule $T$ of $X$. This implies that $X$ is contained in $\operatorname{soc}\left(R_{R}\right)$. Now it is immediate that $\operatorname{soc}\left(\frac{R}{V_{i}}\right)=\frac{\operatorname{soc}\left(R_{R}\right)}{V_{i}}$.

There is some $k$ such that $\operatorname{soc}\left(\frac{A_{k}}{V_{i}}\right)$ is infinitely generated. Also, $\frac{\frac{A_{k}}{V_{i}}}{\operatorname{soc}\left(\frac{A_{k}}{V_{i}}\right)}$ is isomorphic to a direct summand of $\frac{\frac{R}{V_{i}}}{\operatorname{soc}\left(\frac{R}{V_{i}}\right)}$, which, by the preceding paragraph, is isomorphic to $\frac{R}{\operatorname{soc}\left(R_{R}\right)}$. Then, $\frac{\frac{A_{k}}{V_{i}}}{\operatorname{soc}\left(\frac{A_{k}}{V_{i}}\right)}$ is finite dimensional. Also since $\frac{A_{k}}{V_{i}}$ is a finitely generated extending module, this implies, by a standard argument, that $\operatorname{soc}\left(\frac{A_{k}}{V_{i}}\right)$ is finitely generated, a contradiction. Therefore, $\operatorname{soc}\left(R_{R}\right)$ is finitely generated. Along with the fact that $\frac{R}{\operatorname{soc}\left(R_{R}\right)}$ is finite uniform dimensional, this implies that $R_{R}$ has finite uniform dimension. In particular, $R=e_{1} R \oplus \ldots \oplus e_{m} R$, where $\left\{e_{t}: t=1, \ldots m\right\}$ is a complete set of primitive idempotents.

If $m>1$, then $e_{i} R$ are uniform and $R$ clearly satisfies the property $(\mathrm{P})_{r}$, yielding the first part of the theorem.

Now assume that $m=1$. Then $R_{R}$ is indecomposable. If $u \operatorname{dim}\left(R_{R}\right)=1$, then once again $R$ satisfies $(\mathrm{P})_{r}$. So we can assume $\operatorname{udim}\left(R_{R}\right)>1$. Then, for every non-zero right ideal $I$ of $R$, the right $R$-module $\frac{R}{I}$, and all its factors are proper cyclic modules. Invoking Theorem 1 again, $\frac{R}{I}$ has finite uniform dimension. Thus, $R$ is right q.f.d. in 
any case. Now let $L$ be any non-zero nil ideal of $R \cdot \frac{R}{L}$ is a proper cyclic right $R$-module. Since idempotents lift modulo $L$, and $R_{R}$ is indecomposable, $\frac{R}{L}$ must be (right) uniform. Taking $L=N$, the prime radical, we obtain the first part of the last statement. Now assume that $\operatorname{soc}\left(R_{R}\right) \neq 0$. Since $R_{R}$ is indecomposable, every simple right ideal of $R$ is a singular right $R$-module. This implies that $\left[\operatorname{soc}\left(R_{R}\right)\right]^{2}=0$. By the same argument as above, $\frac{R}{\operatorname{soc}\left(R_{R}\right)}$ must be a right uniform ring. This concludes the proof.

COROLLARY 9. A ring satisfying the property $\left(P^{\prime}\right)_{r}$ is right q.f.d.

Proof. By Theorems 2 and 6.

Since an extending module with finite Goldie dimension is a direct sum of uniform modules, the next corollary now follows immediately.

COROLLARY 10. The following statements are equivalent for a ring $R$ :

(i) $R$ has the property $\left(P^{\prime}\right)_{r}$,

(ii) Every proper cyclic right $R$-module is a direct sum of quasi-continuous modules,

(iii) Every proper cyclic right $R$-module is a direct sum of uniform modules.

The next example shows that case (ii) of Theorem 6 is indeed possible.

Example 9. Let $K$ be a field, $R$ be as in Example 5, and $S$ be the subring of $R$ consisting of all elements of the form $\left(\begin{array}{ll}a & b \\ 0 & a\end{array}\right)$, where $a \in K$ and $b \in K^{2}$. Then $S$ is a local ring with property $\left(\mathrm{P}^{\prime}\right)_{r}$. Clearly, $S$ does not have the property $(\mathrm{P})_{r}$.

Also note that $\left(\mathrm{P}^{\prime}\right)_{r}\left(\right.$ even $\left.(\mathrm{P})_{r}\right)$ does not imply $\left(\mathrm{P}^{\prime}\right)_{l}$ : In Example 2, $R e_{22}$ is a proper cyclic left $R$-module which is local but not uniform.

THEOREM 7. Let $R$ be a ring with the property $\left(Q^{\prime}\right)_{r}$. Then, $R$ satisfies one of the following conditions:

(i) $R$ has the property $(Q)_{r}$,

(ii) $R$ is a local right PCQI-ring, or

(iii) $R$ is a semiprime right q.f.d. ring such that $R_{R}$ is indecomposable. In this case, if $R$ is not prime then $J=0$.

In all cases, non-zero prime ideals are maximal.

Proof. We will show the last statement first: Let $P$ be a non-zero prime ideal of $R$. Then $\frac{R}{P}$ is a ring satisfying $(\mathrm{Q})_{r}$. Then by Corollary $5, \frac{R}{P}$ is simple Artinian. Thus, $P$ is a maximal ideal.

By assumption and Theorem 6, we have the following possible cases: $R$ has $(\mathrm{P})_{r}$, or $R_{R}$ is indecomposable and $u \operatorname{dim}\left(R_{R}\right)>1$.

If $R$ has $(\mathrm{P})_{r}$ and $u \operatorname{dim}\left(R_{R}\right)>1$, then $R$ obviously satisfies $(\mathrm{Q})_{r}$. In all other cases, $R_{R}$ is indecomposable (including when $R$ has $(\mathrm{P})_{r}$ and $\left.\operatorname{udim}\left(R_{R}\right)=1\right)$ and q.f.d. So, assume that $R_{R}$ is indecomposable. If the prime radical $N \neq 0$, then $\frac{R}{N}$ is a right selfinjective and right uniform ring. Therefore, it is a local ring, whence so is $R$. It is now clear that $R$ is a right PCQI-ring.

Else, assume that $R$ is semiprime and non-prime. Then, by the first paragraph of this proof, all prime ideals of $R$ are maximal. This implies, as in the proof of Corollary 5 (ii), that $N=J=0$. Now the proof is complete.

One may consider, as in the remark at the end of Section 5 , what would happen if the assumption quasi-injective in the condition $\left(\mathrm{Q}^{\prime}\right)_{r}$ were replaced by continuous, whether 
the new condition would be equivalent to $\left(\mathrm{Q}^{\prime}\right)_{r}$, etc. Such questions ultimately lead to the following general question, which we have been unable to establish in this paper: How to completely characterise the classes of rings satisfying the respective conditions that every (proper) cyclic right module is a direct sum of extending (equivalently quasicontinuous), continuous and quasi-injective modules.

ACKNOWLEDGEMENTS. The first and the third authors acknowledge the support they received from TUBITAK (The Scientific and Technological Research Council of Turkey) for their visit to the Ohio University Center of Ring Theory and its Applications. Part of this work was done during that visit. All authors would like to thank the Center and its members for the warm hospitality they received. Finally, we are greatly indebted to the referee for his/her careful reading of the manuscript and for making very helpful suggestions that have improved the paper.

\section{REFERENCES}

1. J. Ahsan, Rings all of whose cyclic modules are quasi-injective, Proc. London Math. Soc., 27(3) (1973), 425-439.

2. J. Ahsan, On rings with quasi-injective cyclic modules, Proc. Edinb. Math. Soc., II Ser. 19 (1974), 139-145.

3. N. V. Dung, D. V. Huynh, P. F. Smith and R. Wisbauer, Extending modules, Pitman Res. Notes Math. Ser. 313 (1994).

4. N. Er, Rings whose modules are direct sums of extending modules, Proc. Amer. Math. Soc. 137(7) (2009), 2265-2271.

5. A. Facchini, Module theory Birkhäuser, Vol. 167 (Basel, Boston, Berlin, 1998).

6. C. Faith, Algebra II, Ring Theory, Vol. 191 (Springer Verlag, Berlin, New York, 1976).

7. K. R. Fuller, Rings of left invariant module type, Comm. Algebra. 6(2) (1978), 153-167.

8. D. V. Huynh, S. K. Jain and S. R. López-Permouth, When is a simple ring Noetherian?, J. Algebra. 184 (1996), 786-794.

9. S. K. Jain, S. Singh and A. K. Srivastava, On $\Sigma-q$ rings, J. Pure Appl. Algebra. 213 (2009), 969-976.

10. S. K. Jain, S. Singh and R. G. Symonds, Rings whose proper cyclic modules are quasiinjective, Pacific J. Math. 67 (1976), 461-472.

11. R. E. Johnson and E. T. Wong, Quasi-injective modules and irreducible rings, J. London Math. Soc. 36 (1961), 260-268.

12. G. B. Klatt and L. S. Levy, Pre-self injective ringss, Trans. Amer. Math. Soc. 137 (1969), 407-419. $51-55$.

13. A. Koehler, Rings with quasi-injective cyclic modules, Quarterly J. Math. 25 (1974), 1999).

14. T. Y. Lam, Lectures on modules and rings (Springer, Berlin, Heidelberg and New York,

15. B. Osofsky and P. F. Smith, Cyclic modules whose quotients have all complement submodules direct summands, J. Algebra 139 (1991), 342-354.

16. B. Osofsky, Rings all of whose finitely generated modules are injective, Pacific J. Math. 14 (1964), 645-650.

17. B. Osofsky, Noncyclic injective modules, Proc. Amer. Math. Soc. 19 (1968), 1383-1384.

18. G. Puninski, Serial Rings (Kluwer Pub, Netherlands, 2001).

19. S. Singh, Indecomposable modules over Artinian right serial rings in Advances in Ring Theory, (Jain, S. K. and Rizvi, S. T. Editors) (Birkhäuser Verlag, Boston, 1997), 295-304. 